Marek Nalos

Stephen Huang

Ronald Sluyter

Alamgir Khan

Brigitte Santner-Nanan

Ralph Nanan

Anthony S. McLean

\section{"Host tissue damage" signal ATP impairs IL-12 and IFN $\gamma$ secretion in LPS stimulated whole human blood}

Published online: 11 July 2008

(C) Springer-Verlag 2008

The online version of the original article can be found under doi: 10.1007/s00134-008-1156-y.

M. Nalos (®) · S. Huang · A. S. McLean

Department of Intensive Care, Nepean Hospital,

University of Sydney, P.O. Box 63,

Penrith, NSW 2750, Australia

e-mail: mareknalos@gmail.com

Tel.: +61-24-7342490

Fax: +61-24-7343134

R. Sluyter

Department of Medicine, Nepean Clinical School,

University of Sydney, P.O. Box 63,

Penrith, NSW 2750, Australia

A. Khan

Australian Proteome Analysis Facility (APAF),

Macquarie University, Level 4, Building F7B,

Research Park Drive, Sydney, NSW 2109, Australia

B. Santner-Nanan

Department of Paediatrics, Nepean Clinical School,

University of Sydney, P.O. Box 63,

Penrith, NSW 2750, Australia

R. Nanan

Department of Pediatrics, Nepean Hospital, University of Sydney, P.O. Box 63, Penrith, NSW 2750, Australia

\section{Erratum to: Intensive Care Med Dol 10.1007/ s00134-008-1156-y}

Due to an unfortunate oversight the following abstract and keywords were not included in the article.
Abstract Objective: Critical illness is associated with tissue damage, inflammation and the development of immune dysfunction. Leukocyte reprogramming occurs leading to insufficient production of pro-inflammatory cytokines upon subsequent stimulation. Cellular nucleotides released during tissue damage act via purinergic receptors to modulate immune function. We hypothesized that extracellular nucleotides in concentrations similar to those found near injured and ischemic tissues will modulate cytokine secretion. Design and participants: Bench study in 28 healthy human volunteers using standardized lipopolysaccharide (LPS) stimulated ex vivo whole blood cultures (ILCS ${ }^{\circledR}$ ). Setting: The Nepean Hospital Laboratories, University of Sydney. Interventions: Nucleotides ATP, ADP and other P2 purinergic receptor agonists ATP $\gamma$ S, BzATP, UTP and P1 agonist CV1808 were injected into the ILCS $^{\circledR}$, and cultured for 6,12 and $24 \mathrm{~h}$ as indicated. Measurements and results: ATP $(100 \mu \mathrm{M})$ reduced the LPS stimulated secretion of TNF $\alpha$ at 6 and 12 $\mathrm{h}$, as well as IL-12(p70) and IFN $\gamma$ at $24 \mathrm{~h}$ of incubation. ADP, ATP $\gamma$ S, BzATP, and CV1808, but not UTP displayed IL-12(p70) and IFN $\gamma$ reducing effect similar to ATP. Higher ATP concentration $(500 \mu \mathrm{M})$ had even more pronounced immunosuppressive effect. Nucleotides had variable effect on stimulated IL-10 secretion. Conclusions: ATP and ADP at high-micromolar concentrations reduce secretion of the main Th1 cytokines TNF $\alpha$, IL-12(p70) and IFN $\gamma$ in LPS stimulated human blood. As immune dysfunction associated with critical illness is characterized by decreased TNF $\alpha, \mathrm{IL}-12$ and IFN $\gamma$ production by leukocytes, extracellular nucleotides might contribute to its pathogenesis.

Keywords Adenosine triphosphate - Adenosine diphosphate - Sepsis · Endotoxin · Purinergic receptors 